\title{
Human Leukocyte Antigen Genotype as a Marker of Multiple Sclerosis Prognosis
}

\author{
Andreas P. Lysandropoulos, Gaetano Perrotta, Thibo Billiet, Annemie Ribbens, \\ Renaud Du Pasquier, Caroline Pot Kreis, Pietro Maggi, Marie Théaudin
}

\begin{abstract}
Objective: In a previous pilot monocentric study, we investigated the relation between human leukocyte antigen (HLA) genotype and multiple sclerosis (MS) disease progression over 2 years. HLA-A*02 allele was correlated with better outcomes, whereas HLA-B*07 and HLA-B*44 were correlated with worse outcomes. The objective of this extension study was to further investigate the possible association of HLA genotype with disease status and progression in MS as measured by sensitive and complex clinical and imaging parameters. Methods: Hundred and forty-six MS patients underwent HLA typing. Over a 4-year period of follow-up, we performed three clinical and magnetic resonance imaging (MRI) assessments per patient, which respectively included Expanded Disability Status Scale, Multiple Sclerosis Severity Scale, Timed-25-Foot-Walk, 9-Hole Peg Test, Symbol Digit Modalities Test, Brief Visual Memory Test, California Verbal Learning Test-II, and whole-brain atrophy, fluid-attenuated inversion recovery (FLAIR) lesion volume change and number of new FLAIR lesions using icobrain. We then compared the clinical and MRI outcomes between predefined HLA patient groups. Results: Results of this larger study with a longer follow-up are in line with what we have previously shown. HLA$\mathrm{A} * 02$ allele is associated with potentially better MS outcomes, whereas HLA-B*07, HLA-B*44, HLA-B*08, and HLA-DQB $1 * 06$ with a potential negative effect. Results for HLA-DRB $1 * 15$ are inconclusive. Conclusion: In the era of MS treatment abundance, HLA genotype might serve as an early biomarker for MS outcomes to inform individualized treatment decisions.
\end{abstract}

RÉSUMÉ: Le génotype des antigènes leucocytaires humains comme marqueur de l'évolution de l'état de santé de patients atteints de sclérose en plaques. Objectif : Dans une étude pilote monocentrique menée précédemment, nous nous sommes intéressés au lien existant entre le génotype des antigènes leucocytaires humains (ou « HLA » en anglais) et l'évolution de l'état de santé de patients atteints de sclérose en plaques (SP) au cours d'une période de deux ans. Alors que nous avons pu établir une corrélation entre l'allèle HLA-A*02 et une évolution favorable de l'état de santé de patients atteints de SP, il nous a été possible d'associer les allèles HLA-B*07 et HLA-B*44 à une évolution défavorable. En cela, l'objectif de cette étude complémentaire a consisté à étudier de façon plus approfondie l'association possible entre le génotype des HLA et l'évolution de la SP telle que mesurée par des paramètres cliniques et des paramètres d'IRM à la fois complexes et sensibles. Méthodes : Au total, 146 patients atteints de SP ont été soumis à une analyse de typage de leurs HLA. Au terme d'un suivi de 4 ans, nous avons effectué 3 examens cliniques et d'imagerie pour chaque patient. Outre l'atrophie totale du cerveau et des changements au volume et au nombre de nouvelles lésions détectées, à l'aide du logiciel icobrain, par la technique d'IRM dite «FLAIR » (fluid-attenuated inversion recovery), nous avons ainsi tenu compte des méthodes et paramètres suivants : EDSS, MSSS, T25FW, 9-HPT, SDMT, BVMT et CVLT-II. Au moyen d'examens d'IRM et d'un point de vue clinique, nous avons alors comparé entre eux des groupes de patients HLA prédéfinis en ce qui regarde l'évolution de leur état de santé. Résultats : Les résultats de cette étude plus vaste dont la période de suivi a été plus longue corroborent ce que nous avions précédemment décrit. L'allèle HLA-A*02 a en effet été associée à une évolution plus favorable de l'état de santé de patients atteints de SP tandis que les allèles HLA-B*07, HLA-B*44, HLA-B*08 et HLA-DQB1*06 ont entraîné un impact potentiellement négatif. Mentionnons aussi qu'il nous a été impossible de tirer des conclusions en ce qui concerne l'allèle HLA-DRB1*15. Conclusion : À une ère où les traitements contre la SP sont légions, il se peut que le génotype des HLA puisse être utilisé à titre de biomarqueur précoce de l'évolution de cette maladie, et ce, en vue d'éclairer les décisions prises en matière de traitements individualisés.

Keywords: HLA genotype, Multiple sclerosis, Prognostic, Magnetic resonance imaging, Clinical score doi:10.1017/cjn.2019.329

Can J Neurol Sci. 2020; 47: 189-196

\section{INTRODUCTION}

The genetic risk for multiple sclerosis (MS) is related to a series of HLA class II and I alleles. ${ }^{1-3}$ HLA-DRB1*15:01 allele has been shown to have the strongest association with MS, especially in Caucasian populations. ${ }^{1,2,4-7}$ HLA class I alleles have been associated with either reduced (HLA-A*02:01, HLA-B*44:02) $)^{8-12}$ or increased (HLA-A*03, HLA-B*07) susceptibility to MS. ${ }^{10,11,13,14}$

There is an unmet need for a biomarker with prognostic value in MS. Limited studies with contradictory results have

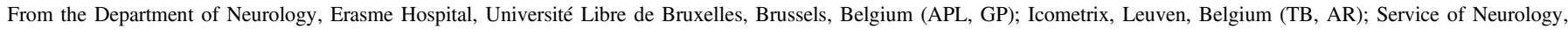
Department of Clinical Neurosciences, Lausanne University Hospital and University of Lausanne, Lausanne, Switzerland (RDP, CPK, PM, MT)

Received July 25, 2019. Final Revisions Submitted November 7, 2019. Date of Acceptance November 23, 2019.

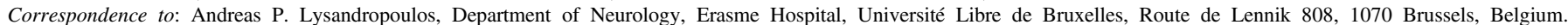
Email: andreas.lys@hotmail.com 


\section{Table 1: Description of the patient population at baseline}

\begin{tabular}{l|c}
\hline \# patients & 146 (117 Erasme, 29 CHUV) \\
\hline Median age, year (range) & $41.1 \pm 11.2(16.95-67.88)$ \\
\hline Mean disease duration, year (range) & $11.5 \pm 6.7(1.75-38.05)$ \\
\hline Median EDSS (range) & $2.4 \pm 1.3(1-6.5)$ \\
\hline Gender \# (\%) patients) & Males 44 (30.1), females 102 (69.9) \\
\hline MS types $(n)$ & 132 RR-MS, 8 SP-MS, 6 PP-MS \\
\hline On treatment (RRMS and SPMS) $(n, \%)$ & 93.8 \\
\hline First line* & $37.0 \%$ \\
\hline Second line* & $56.8 \%$ \\
\hline
\end{tabular}

EDSS = expanded disability status scale; RRMS = relapsing remitting MS; SPMS = secondary progressive MS.

*As per EMA definition. All patients remained in the respective treatment line throughout the study period.

investigated possible association of HLA genotype with MS severity

by evaluating few and poorly sensitive clinical and magnetic resonance imaging (MRI) parameters. ${ }^{15-22}$

In a previous study, we reported on the relationship between HLA genotype and MS disease progression over a 2-year period, regarding both clinical including Expanded Disability Status Scale (EDSS) and Multiple Sclerosis Severity Scale (MSSS) and MRI outcomes (new lesion count and brain volume). We found that the HLA-A*02 allele was associated with better clinical and MRI outcomes, whereas the HLA-B*07 and HLA-B*44 alleles with a global negative effect on disease status. Results for the HLA-DRB1*15, HLA-DQB1*06, and HLA-B*08 alleles were inconclusive. The influence of confounding variables, such as age, gender, disease duration, MS type and treatment, scanner model, MRI field strength, and gadolinium (Gd) enhancement on the statistical analysis was limited. ${ }^{23}$

In this study, we aim to further explore the possible association between HLA genotype and MS progression by incorporating data from an additional MS center as well as by evaluating the patients over a longer period of time (3 time points over a mean follow-up of 4 years).

\section{Materials ANd Methods}

\section{Patients}

We included patients with relapsing remitting (RR), secondary progressive (SP), or primary progressive (PP) MS followed in two MS centers: the Lausanne University Hospital, Lausanne (Switzerland) and the Erasme University Hospital, Brussels (Belgium). An overview of patients' characteristics is provided in Table 1.

\section{HLA Typing}

HLA typing was performed on DNA extracted from peripheral blood mononuclear cells by low- to intermediate-resolution polymerase chain reaction using sequence-specific oligonucleotides. Reverse dot blotting was carried out on a nylon membrane containing immobilized sequence-specific oligonucleotide probes used for the typing of HLA class I (HLA-A*02, HLA-B*07,
Table 2: Overview of MRI and clinical variables

\begin{tabular}{l|l}
\hline Clinical scores & $\begin{array}{l}\text { EDSS at } 3 \text { time points and change over } \\
\text { time (all patients) }\end{array}$ \\
\cline { 2 - 2 } $\begin{array}{l}\text { MSSS at } 3 \text { time points and change over } \\
\text { time (all patients) }\end{array}$ & $\begin{array}{l}\text { 9HPT at } 3 \text { time points and change over } \\
\text { time (Erasme patients) }\end{array}$ \\
\cline { 2 - 2 } & $\begin{array}{l}\text { T25FW at } 3 \text { time points and change over } \\
\text { time (Erasme patients) }\end{array}$ \\
\cline { 2 - 3 } & $\begin{array}{l}\text { SDMT at } 3 \text { time points and change over } \\
\text { time (Erasme patients) }\end{array}$ \\
\cline { 2 - 3 } & BVMT-R at baseline (Erasme patients) \\
\cline { 2 - 3 } & CVLT at baseline (Erasme patients) \\
\hline \multirow{2}{*}{ MRI scores } & $\begin{array}{l}\text { Whole brain volume at 3 time points and } \\
\text { change over time (all patients) }\end{array}$ \\
\cline { 2 - 3 } & $\begin{array}{l}\text { Lesion volume at 3 time points and } \\
\text { change over time (all patients) }\end{array}$ \\
\cline { 2 - 3 } & New lesion count (all patients) \\
\hline
\end{tabular}

BVMT-R = Brief Visual Memory Test; CVLT-II = California Verbal Learning Test-II; EDSS = Expanded Disability Status Scale; MSSS = Multiple Sclerosis Severity Scale; PASAT-3 = Paced Auditory Serial Addition Test; SDMT $=$ Symbol Digit Modalities Test; T25FW= Timed-25-Foot-Walk; 9-HPT = 9-Hole Peg Test.

HLA-B*44) (all patients) and HLA class II (HLA-DRB1*15, HLA-DRB1*04, HLA-DRB1*07, HLA-DQB1*06) alleles (Erasme patients) (INNO-LiPA ${ }^{\circledR}$, Fujirebio).

\section{Clinical and MRI Evaluation}

We assessed the patients at 3 time points over a 4-year period by evaluating various clinical and MRI parameters (Table 2). Data were collected from patients' medical records. All clinical and MRI evaluations were performed as part of routine practice. Clinical tests were done at the same time as the MRI. The mean interval in days between first and second evaluation was $823.52 \pm 382.52$ and from second to the third was $636.68 \pm 241.90$.

\section{Clinical Evaluation}

Annualized percentage of change (apc) in EDSS, MSSS, Timed-25-Foot-Walk (T25FW), 9-Hole Peg Test (9HPT), and Symbol Digit Modalities Test (SDMT) was computed, (apc-EDSS, apc-MSSS, apc-T25FW, apc-9HPT, and apc-SDMT, respectively) by means of a linear regression on the available longitudinal results. Progression (Yes/No) in EDSS was defined as an increase by at least 1 point (for baseline EDSS below 6) or at least 0.5 points (for baseline EDSS of 6 or higher), confirmed $\geq 24$ weeks apart. For 9HPT and T25FW, progression was defined as an increase in the time by more than $20 \%$, confirmed $\geq 24$ weeks apart. EDSS plus was defined as progression in either EDSS, 9HPT, or T25FW, confirmed $\geq 24$ weeks apart.

\section{MRI Measurements}

Scans obtained from clinical routine included a fluid-attenuated inversion recovery (FLAIR) sequence and a T1-weighted turbo field echo sequence (pre- or post-Gd injection). All MRI scans 
Table 3: Overview of HLA groups assessed in this study

\begin{tabular}{|c|}
\hline $\mathrm{B} * 07+$ vs. $\mathrm{B} * 07$ \\
\hline DRB1*15-/B*07+ vs. DRB1*15-/B*07- \\
\hline DRB $1 * 15+/ B * 07+$ vs. DRB $1 * 15+/ B * 07-$ \\
\hline $\mathrm{A} * 02-/ \mathrm{B} 7+$ vs. all others \\
\hline $\mathrm{A}^{*} 02+$ vs. $\mathrm{A}^{*} 02-$ \\
\hline DRB $1 * 15-/ A * 02+$ vs. DRB $1 * 15-/ A * 02-$ \\
\hline DRB $1 * 15+/ A * 02+$ vs. DRB $1 * 15+/ A * 02-$ \\
\hline DRB1*15+ vs. DRB1*15- \\
\hline B*08+ vs. B*08- \\
\hline$B * 44+$ vs. $B * 44-$ \\
\hline DQB1*06+ vs. DQB1*06- \\
\hline DQB1*06+DRB $1 * 15+$ vs. all others \\
\hline
\end{tabular}

were processed using icobrain ms (https://icometrix.com/products/ icobrain-ms). ${ }^{24-26}$ The method analyzes two consecutive scans simultaneously, yielding robust and consistent measurements for whole brain volume and lesion load (volume and count). To obtain an overall atrophy score allowing multiple time points, a linear fit was applied on the whole brain volumes, and the annualized percentage brain volume change (aPBVC) was computed between first and last time point. In the same way, an annualized percentage in lesion volume change (aPLVC) was computed. The number of new lesions was summed to obtain the overall new lesions count since baseline.

Progression (Yes/No) in whole brain volume decrease was defined as aPBVC stronger than $-0.4 \%$. To define progression in lesion count, a threshold was set to a minimal new lesion size of $5 \times 3 \mathrm{~mm}$ on $3 \mathrm{D}$ images.

\section{Statistical Analysis}

The relationship between HLA genotype and clinical and MRI outcomes was evaluated by comparing group of patients with different HLA genotypes. Based on literature reports from previous studies, ${ }^{12,27,28}$ the analysis focused on specific and potentially relevant subgroups of HLA alleles: HLA-A*02, HLA-B*07, HLA-B*44, HLA-B*08, HLA-DRB $1 * 15$, and HLA-DQB $1 * 06$ alleles, and their combinations (Table 3).

Thus, the statistical analyses evaluated differences in clinical scores and MRI measurements between patients from each HLA group and its counterpart. The statistical analysis followed three major steps: (1) an individual assessment of the different clinical scores and MRI measurements describing the disease status and progression with respect to the predefined HLA groups, (2) an overall assessment of the relation between MRI/clinical outcomes and specific HLA genotypes, (3) an assessment of the influence of diverse covariates (confounding variables) : gender, age, disease duration, MS type, and treatment (first- vs. second-line), scanner type (1.5 T vs. $3 \mathrm{~T}$ scanner), and Gd injection.

First, all data were evaluated to comply with the normality assumptions required for parametric statistical tests. The distribution of all variables was checked based on scatter plots and additionally normality was addressed based on the Shapiro-Wilk test. To gain normality, T2 lesion volume was cube root transformed, and 9-HPT and T-25FW scores were logarithmically (base 10) transformed.

Dependent variables for which multiple time points were available (whole brain volume, lesion volume, EDSS, MSSS, T25FW, 9HPT, SDMT) were analyzed using linear mixed effects modeling (lmer function as implemented in the lme4 package in $\mathrm{R})$. This allows for the joint analysis of patients with two or three scans. The standard model included the interaction between time (tp1, tp2, or tp3) and HLA group, and their main effects as fixed effects. A random effect was added to account for the repeated measures per patient. Dependent variables for which only one measurement per patient was assessed (aPBVC, aPLVC, total new lesions count, apc-EDSS, apc-MSSS, apc-T25FW, apc9HPT, apc-SDMT, California Verbal Learning Test-II, Brief Visual Memory Test-Revised (BVMT-R)) were analyzed using a linear model with HLA group as fixed effect. From all models, the main effect of group was assessed using a type 2 ANOVA and was considered significant for $p<0.05$. To obtain an overall impression of protectiveness of a certain HLA allele across clinical and MRI outcomes, we computed the weighted average of test statistics from the individual ANOVA. The T-statistics for main effect of group (in absence of a covariate) were multiplied by +1 or -1 to yield a positive value in case of a protective effect of the first of both groups. The weighted average across outcomes is then suggestive for an overall beneficial, adverse, or neutral effect of a certain HLA allele's presence.

Each covariate's influence on a clinical or MRI measurement was tested by adding it to the model of interest (in absence of other covariates). Then the type 2 ANOVA analysis was repeated, and the significance of the main effect of group was reconsidered.

For progression markers, the odds ratio (OR) was computed for the different HLA groups, and progression was statistically compared between groups using the two-sided Fisher's exact test on the contingency table $(p<0.05)$. Similar to the continuous outcomes, an overall impression of protectiveness can be given based on progression outcomes. To do so, the deviation from equal odds (i.e. 1-OR) was computed, after which a positive deviation is again indicative for a beneficial effect for the first mentioned group.

\section{RESULTS}

Table 4 provides the overview of the effects of HLA genotypes on clinical and MRI parameters. An important note is that the T-statistics in this table should only be compared across (rows) and not between columns (group comparisons), because of different sample sizes. Furthermore, because not all clinical measures have been evaluated in all participants (as is the case for the MRI variables), the T-statistics of these clinical variables are also less comparable among each other. For this reason, Table 4 includes a separate average for the clinical and MRI measurements. Given the exploratory nature of this study, no correction for multiple comparisons was applied. Therefore, in the following paragraphs describing each of the HLA subtypes, the patterns of findings should be interpreted rather than the exact statistical results.

\section{HLA-B*07}

The evaluation relied on 45 patients harboring the HLA-B*07 allele and 101 patients without this allele. Both groups were 
Table 4: Weighted T-statistics of the main effect of group, in absence of covariates. A positive T-statistic indicates a protective effect of the first group. A negative T-statistic indicates increased susceptibility of the first group. A positive deviation from equal odds ratio indicates less probability of the first group to undergo progression

\begin{tabular}{|c|c|c|c|c|c|c|c|c|c|c|c|c|}
\hline & B7+ vs. B7- & $\begin{array}{c}\text { DRB15-/B7+ vs. } \\
\text { DRB15-/B7- }\end{array}$ & $\begin{array}{c}\text { DRB15+/B7+ } \\
\text { vs. DRB15+/ } \\
\text { B7- }\end{array}$ & $\begin{array}{l}\text { A2-/B7+ vs. } \\
\quad \text { rest }\end{array}$ & A2+ vs. A2- & $\begin{array}{c}\text { DRB15-/A2+ } \\
\text { vs. DRB15-/ } \\
\text { A2- }\end{array}$ & $\begin{array}{c}\text { DRB15+/A2+ } \\
\text { vs. DRB15+/ } \\
\text { A2- }\end{array}$ & $\begin{array}{l}\text { DRB15+ vs. } \\
\text { DRB15- }\end{array}$ & B8+ vs. B8- & B44+ vs. B44- & $\begin{array}{l}\text { DQ6+ vs. } \\
\text { DQ6- }\end{array}$ & $\begin{array}{l}\text { DQ6+DR15+ } \\
\text { vs. rest }\end{array}$ \\
\hline \multicolumn{13}{|c|}{ Two-sample T-statistic } \\
\hline $\begin{array}{l}\text { Norm. whole brain } \\
\text { volume }\end{array}$ & 0.15 & 1.00 & -0.27 & 0.59 & 0.16 & 1.46 & -0.80 & -0.36 & $-2.07^{* * *}$ & 1.43 & -0.37 & -0.40 \\
\hline aPBVC & $-1.82 *$ & -0.71 & -1.38 & -0.01 & -0.99 & -0.07 & -1.52 & -0.37 & 0.38 & -0.51 & -1.04 & -0.36 \\
\hline Lesion volume & $2.11^{* *}$ & $1.69^{*}$ & 0.58 & 0.54 & 0.84 & 0.72 & -1.14 & 0.21 & -0.71 & $2.11 *$ & 0.66 & 0.17 \\
\hline aPLVC & 0.97 & 0.58 & 1.36 & $1.69 *$ & -0.65 & -0.78 & 0.10 & 0.55 & -0.75 & $-1.78^{*}$ & -0.03 & 0.57 \\
\hline $\begin{array}{l}\text { Total new lesions } \\
\text { count }\end{array}$ & 1.09 & 0.04 & -0.54 & 0.34 & 0.25 & 0.31 & -0.52 & $1.83^{*}$ & 0.93 & $-3.08^{* * *}$ & 0.38 & $1.90^{*}$ \\
\hline BVMT-R & 0.37 & 0.39 & 0.16 & -0.33 & 1.20 & $1.78^{*}$ & -0.29 & 0.11 & -1.43 & -0.45 & 0.90 & 0.14 \\
\hline CVLT-II & -1.21 & -0.86 & -0.86 & -0.98 & -0.05 & 0.55 & -0.64 & -0.30 & -0.19 & -0.55 & 1.12 & -0.46 \\
\hline EDSS & -1.02 & 0.00 & -1.02 & -1.57 & $1.72 *$ & 1.11 & 1.46 & -1.52 & -0.47 & 0.68 & -1.49 & -1.58 \\
\hline Apc-EDSS & 1.21 & -0.17 & 0.22 & 0.31 & 0.58 & 0.11 & -0.29 & 0.91 & $-2.54^{* * *}$ & 0.56 & 0.68 & 0.88 \\
\hline MSSS & -0.60 & -0.30 & -1.18 & -1.38 & $2.03^{* * *}$ & 1.12 & 1.57 & -1.01 & 0.61 & -0.39 & -1.74 & -1.22 \\
\hline Apc-MSSS & 1.09 & -0.18 & 0.38 & 0.02 & 1.24 & 0.43 & 0.17 & 0.12 & $-2.48^{* * *}$ & 0.74 & 0.04 & 0.09 \\
\hline SDMT & 0.10 & -0.66 & 0.71 & 0.05 & -0.25 & 0.18 & -0.59 & -0.23 & -1.64 & -0.31 & -0.02 & -0.36 \\
\hline Apc-SDMT & -0.33 & 1.18 & -1.45 & 0.18 & -0.68 & -0.85 & 0.06 & -0.20 & 1.05 & -0.31 & -0.72 & 0.18 \\
\hline $\mathrm{T} 25 \mathrm{FW}$ & 0.20 & 1.12 & -0.16 & -0.65 & $1.69^{*}$ & 1.29 & 1.08 & -0.42 & -0.44 & 0.17 & -0.46 & -0.41 \\
\hline Apc-T25FW & -1.12 & -1.17 & -0.35 & -1.14 & 0.40 & -0.11 & 0.80 & -0.60 & -0.25 & 0.24 & $-1.83^{*}$ & -0.75 \\
\hline 9HPT & 0.10 & 0.70 & -0.49 & 0.18 & 0.33 & 0.53 & -0.19 & -0.06 & -0.49 & -0.25 & -0.49 & 0.10 \\
\hline Аpc-9HРT & -0.20 & -1.14 & -0.04 & 0.32 & -0.16 & -0.57 & 0.74 & 1.09 & $1.77 *$ & $-2.06^{* * *}$ & 0.27 & 1.02 \\
\hline Average MRI & 0.28 & 0.46 & -0.18 & 0.57 & -0.13 & 0.39 & -0.84 & 0.25 & -0.51 & -0.23 & -0.19 & 0.25 \\
\hline Average clinical & -0.16 & -0.11 & -0.34 & -0.45 & 0.66 & 0.56 & 0.21 & -0.16 & -0.58 & -0.21 & -0.12 & -0.19 \\
\hline Average all & -0.06 & 0.01 & -0.30 & -0.22 & 0.48 & 0.53 & -0.02 & -0.07 & -0.57 & -0.21 & -0.14 & -0.09 \\
\hline \multicolumn{13}{|c|}{ Deviation from equal progression odds ratio } \\
\hline Atrophy & -0.23 & 0.05 & -0.48 & 0.04 & 0.04 & 0.21 & -0.51 & 0.18 & -0.23 & -0.44 & 0.04 & 0.16 \\
\hline New $5 \mathrm{~mm}$ lesion & 0.41 & 0.16 & -0.02 & 0.72 & -0.21 & 0.44 & -0.82 & $0.81^{* * *}$ & 0.74 & -1.41 & -0.19 & $0.80^{\text {*** }}$ \\
\hline EDSS prog & 0.02 & -0.35 & 0.35 & -0.36 & 0.38 & 0.46 & 0.02 & -0.38 & -0.82 & 0.24 & -0.38 & -0.44 \\
\hline EDSS plus & -0.39 & -0.78 & -0.28 & -0.26 & 0.22 & 0.24 & 0.19 & -0.07 & -0.09 & 0.19 & -0.17 & -0.10 \\
\hline T25FW prog & -0.83 & -1.34 & -0.62 & -0.91 & 0.44 & 0.59 & 0.24 & -0.25 & 0.03 & -0.07 & -0.61 & -0.31 \\
\hline 9HPT prog & -0.17 & -0.49 & -0.66 & 0.52 & -0.28 & -0.42 & -0.07 & 0.34 & $1.00^{* * *}$ & 0.06 & 0.07 & 0.32 \\
\hline
\end{tabular}

9HPT prog

$* p<0.1$.

$* * p<0.05$.

$* * * p<0.01$. 
Table 5: Summary of general trends of the statistical analysis of the relationship between HLA genotype and clinical/MRI outcomes

\begin{tabular}{|c|c|c|c|c|c|c|}
\hline & DRB15 & B7 & $\mathbf{A 2}$ & DQ6 & B8 & B44 \\
\hline Effect & inconclusive & Toward negative & Protective & Toward negative & Toward negative & Toward negative \\
\hline \multirow{4}{*}{$\begin{array}{l}\text { Significant scores } *= \\
\text { became or remained } \\
\text { significant after } \\
\text { controlling for } \\
\text { covariates }\end{array}$} & \multirow[t]{4}{*}{ New lesions odds ratio } & aPBVC* & \multirow[t]{4}{*}{ MSSS } & \multirow[t]{4}{*}{ Apc-T25FW* } & Brain volume & New lesions count \\
\hline & & \multirow[t]{3}{*}{ Lesion volume } & & & Apc-EDSS* & aPLVC* \\
\hline & & & & & Apc-MSSS* & 9HPT* \\
\hline & & & & & Арc-9HРТ* $*$ & apc-9HPT \\
\hline
\end{tabular}

$* p<0.05$.

balanced in terms of age, gender, and disease duration. Global T-statistic pointed toward a negative impact of the HLA-B*07 allele. A trend toward a negative effect was observed for aPBVC indicating more severe whole-brain atrophy for HLA-B*07. After controlling for MS type, this effect became significantly different $(t=-1.98, p=0.05)$. The FLAIR lesion volume was found to be significantly lower in the HLA-B*07 group $(t=-2.11$, $p=0.029$ ). This finding was however not robust for controlling against scanner model, contrast, field strength, patient sex, age, treatment line, MS type, or MS duration (all $p>0.05$ ).

With regard to disease progression, presence of HLA-B*07 allele appeared to have a negative effect on most clinical scores and whole-brain atrophy.

Similar findings were obtained when the effect of the HLA$\mathrm{B} * 07$ allele was evaluated in the subgroup of patients harboring the HLADRB $1 * 15$ allele. The majority of measurements pointed toward a negative effect of HLA-B*07.

\section{HLA-A *02}

The evaluation of the HLA-A*02 allele was based on a group of $64 \mathrm{MS}$ patients compared to $82 \mathrm{MS}$ patients without HLA-A*02. Both groups were balanced in terms of gender, age, and disease duration. HLA-A*02 seemed to be associated with better prognosis according to the overall scoring based on the averaged T-statistic. This effect was significant $(p<0.05)$ for MSSS, although it did not remain after controlling for treatment line. Furthermore, a trend $(p<0.1)$ for protective effects of HLA-A*02 on EDSS and T25FW was found. Slightly higher brain volume and lower lesion volume were found for patients harboring HLA-A*02 allele, yet also more whole brain volume change and stronger increase in lesion volume were found.

Presence of HLA-A*02 was associated with a trend toward a protective effect for the majority of progression markers.

The effect of the HLA-A*02 allele was also evaluated in the subpopulation of patients harboring the HLA-DRB $1 * 15$ allele. In this analysis, 19 vs. $34 \mathrm{MS}$ patients with and without the HLA-A*02 allele were compared. Both groups were still balanced in terms of gender, age, and disease duration. The global protective effect of HLA-A*02 was not present anymore, even though MSSS, EDSS, and T25FW still leaned toward a protective effect of HLA-A*02. HLA-A*02 had a negative impact on annualized whole-brain atrophy (annualized percentage of brain volume change) in the patients who were also HLADRB $1 * 15$ positive. In contrast to the overall population harboring HLA-A*02, in the subpopulation of HLADRB $1 * 15$ carriers,
HLA-A*02 was associated with higher lesion volume, higher new lesions count, and lower normalized whole brain volume, although not significant without covariates $(p>0.05)$.

Within the subgroup of MS patients without HLA-DRB $1 * 15$, 23 patients had the HLA-A*02 allele and 41 patients did not. Most scores as well as the overall score pointed toward a protective effect. After controlling for age at baseline or MS duration, the increased BVMT-R in the group harboring HLA-A*02 became significant (with age: $t=2.20, p=0.031$, with MS duration: $t=2.31, p=0.024)$. Presence of HLA-A $* 02$ in the absence of HLA-DRB $1 * 15$ had a protective effect for the majority of clinical and MRI markers of progression, although not significant $(p>0.05)$.

\section{Combination HLA-B*07 without HLA-A*02}

As both MS patients with HLA-B*07 and patients without HLA-A*02 seemed to be more prone to MS progression, the combination of both alleles might indicate an even stronger susceptibility. The group of patients with HLA-B*07 and without HLA-A*02 (22 patients) was compared to all other patients (124 patients). Groups were matched in terms of age and disease duration. Global T-statistics and various clinical scores pointed in the direction of a negative effect. However, none of the differences between both groups reached the threshold for significance. Overall, it was not clear whether the effect of HLA-B*07 without HLA-A*02 further amplified the negative overall effect. Presence of HLA-B*07 in the absence of HLA-A*02 tended to have a negative effect on clinical markers of disease progression.

\section{HLA-DRB1 $* 15$}

The effect of this allele was evaluated in a group of $53 \mathrm{MS}$ patients with HLA-DRB $1 * 15$ and 64 MS patients without. Both groups were comparable with respect to age, gender, and disease duration. The effect of HLA-DRB $1 * 15$ allele was unclear. The global T-statistic was marginally negative and most individual scores for clinical and MRI outcomes were negative. Presence of HLA-DRB $1 * 15$ had a positive effect on some MRI markers of disease progression. In particular, from the OR analysis, it became clear that patients harboring the HLA-DRB $1 * 15$ allele were about 5 times less likely to get new lesions at follow-up $(\mathrm{OR}=0.19$, $p=0.02)$. On the other hand, lower brain volume and worse whole-brain atrophy were found for HLA-DRB $1 * 15$ carriers, yet not significant.

Patients with and without HLA-DRB1*15 were also used for the analyses of subpopulations (e.g. see analyses of 
HLA-A*02 and HLA-B*07), which showed no clear effect of HLA-DRB $1 * 15$.

\section{HLA-B*08}

For HLA-B*08 allele, there was a discrepancy in group size between patients with and without the allele (24 vs. 122 patients). The groups were well matched in terms of age and gender, but slightly imbalanced for disease duration. The overall statistical score pointed toward a negative direction. In absence of any covariates, the HLA-B*08 group had significant lower whole brain volume compared to the rest of the patients (not protective, $t=-2.07, p=0.046$ ), yet not significant after controlling for scanner model, field strength, contrast, patient age, MS duration, sex, or treatment line. In terms of clinical scores, HLA-B*08 had lower apc-EDSS (protective, $t=-2.54$, $p=0.012$ ) and lower apc-MSSS (protective, $t=-2.48$, $p=0.014)$. Both findings remained significant after controlling for single covariates. Furthermore, a trend toward lower apc9HPT was found for HLA-B*08 carriers, which turned significant $(p<0.05)$ when controlling for MS type $(t=-2.48$, $p=0.015)$ or MS duration $(t=-2.01, p=0.047)$.

Effect of HLA-B*08 on disease progression was ambiguous. None of the HLA-B*08 carriers showed progression in the 9HPT score, explaining the significant better $\mathrm{OR}(\mathrm{OR}=0, p=0.03)$. Also, HLA-B*08 carriers were 4 times less likely to develop a new $5 \mathrm{~mm}$ diameter lesion. On the other hand, they more often experienced EDSS and whole-brain atrophy progression (not significant).

\section{HLA-B $* 44$}

Patients with HLA-B*44 seemed to be more susceptible to MS progression. Group sizes between patients with and without HLA-B $* 44$ were different ( 24 vs. 122 patients), but both groups were balanced in terms of gender and disease duration. Patients without HLA-B*44 were slightly older (mean age at baseline of HLA-B $* 44$ carriers 36.3 vs. 42.1-year-old without this allele).

The overall statistical score pointed toward a negative direction. The overall count of new lesions was significantly higher in HLA-B $* 44$ carriers compared to noncarriers $(t=3.08$, $p=0.002$ ), which was still the case after controlling for any covariate. Although there was a trend for smaller total lesion volume in the carriers, the significant larger count of new lesions was paralleled by a trend toward larger increase in total lesion volume (not significant), driving the overall adverse effect of the HLA-B*44 allele. Furthermore, after controlling for age at baseline, an increased aPLVC in HLA-B $* 44$ carriers $(t=2.40$, $p=0.018$ ) was found, indicative for vulnerability. We found no significant OR for progression of clinical or MRI outcomes between carriers and noncarriers, although the odds for new lesion count were 2.4 times as high for HLA-B*44 carriers (not significant). Regarding clinical outcomes, there was a larger increase over time in 9HPT duration for HLA-B $* 44(t=2.06$, $p=0.042)$. This remained after controlling for any covariate. Furthermore, the absolute value of 9HPT was found higher in the carrier group (not protective) after controlling for baseline age $(t=1.39, p=0.017)$, MS duration $(t=1.48, p=0.013)$, treatment line $(t=1.02, p=0.029)$, patient sex $(t=1.20, p=0.029)$, or MS type $(t=1.23, p=0.023)$.

\section{HLA-DQB1*06}

The evaluation of HLA-DQB $1 * 06$ was based on a subset of patients, consisting of 71 patients harboring HLA-DQB $1 * 06$ and 46 without this allele. There was a trend toward a negative effect on MRI outcomes on average, mainly because of a trend for stronger atrophy (not significant). Regarding clinical parameters, a trend for increased apc-T25FW (not protective) was found in HLA-DQB $1 * 06$ carriers, yet this only became significant after controlling for MS duration $(t=2.04, p=0.044)$

Presence of HLA-DQB $1 * 06$ had a negative effect on the majority of disease progression MRI and clinical markers.

Results are summarized in Table 5.

\section{DiscuSSION}

This extension study on a bigger sample and with a longer follow-up mainly confirmed the results of our previous work. ${ }^{23}$ The presence of HLA-B*07, HLA-B*08, HLA-B*44, and HLADQB $1 * 06$ was correlated to a worse disease status and/or disease progression as evaluated by multiple clinical and MRI outcomes, whereas HLA-A*02 to a better disease status. Presence of HLADRB $1 * 15$ had an ambiguous effect. Presence of this allele in combination with HLA-A*02 seemed to damp the protective effect of HLA-A*02.

This is the first study to investigate the relation between HLA genotype and overall MS progression by applying many clinical and MRI outcomes and using a validated software for MRI measurements. MS being a heterogeneous disease, multiple disease parameters should be evaluated in order to explore any correlation between a biomarker like HLA genotype and disease progression. The clinical relevance of the potential impact of HLA genotype on each individual clinical parameter is difficult to extrapolate from this study and remains to be explored in bigger cohorts and longer follow-up periods. Our results are in line with prior studies on this subject ${ }^{1,27,28}$ and further strengthen the role of HLA genotype as a potential biomarker of disease trajectory that can be used early in the MS course to inform treatment decisions. There are currently many available drugs for the treatment of MS and yet it is challenging to predict treatment response at the individual level. Therefore, such biomarkers of disease progression could be of great interest to help neurologists in the treatment choice.

MHC class I and II alleles cannot yet be considered as causal variants; they probably represent markers of independent protective haplotypes within the MHC. It is also possible that other alleles in linkage disequilibrium with these HLA markers (HLA-A*02, HLA-B*07, HLA-B*08, HLA-B*44, HLA-DRB $1 * 15)$ could be required to achieve the protective or non-protective effects observed. Several hypotheses have been made to explain the potential causal relationship between specific HLA alleles and MS risk and prognosis, including those related to effect of CD8+ T cells, Vitamin D, and EpsteinBarr virus. ${ }^{18,27-30}$

This study presents some limitations. The large number of statistical tests may require a type 1 error correction. However, given the exploratory nature of this study and, in order to enhance sensitivity, we did not correct for multiple comparisons. Furthermore, not all HLA subtypes were equally prevalent in our sample, leading to differing sample sizes for the 
various evaluations. Some group differences may therefore be underestimated. As a result, results of individual statistical tests should be interpreted with caution. This is why we opted to interpret the pattern of positive or negative weighted $\mathrm{T}$-statistics providing a general idea of the direction of changes and effect size. Another limitation is the lack of MHC Class II data as well as some clinical data from one MS center (CHUV, Lausanne). Finally, as outlined in Table 1, our cohort was predominantly composed of MS patients of the RR type. While we included the MS type as a covariate in statistics, our results may therefore be mostly representative for RR and less for progressive types.

Larger and longer studies with additional potential biomarkers, such as neurofilament in cerebrospinal fluid, optic coherence tomography, are needed to confirm these results. Moreover, the investigation of the potential effect of HLA genotype on treatment response especially in patients who switched from one compound to another would be of interest.

\section{Disclosures}

APL has nothing to disclose.

GP has nothing to disclose.

TB reports personal fees from icometrix, during the conduct of the study; personal fees from icometrix, outside the submitted work.

$\mathrm{AR}$ reports personal fees from icometrix $\mathrm{NV}$, during the conduct of the study; personal fees from icometrix NV, outside the submitted work; In addition, Dr. Ribbens has a patent EP2996085 issued.

RDP reports grants from Biogen, personal fees from Biogen, personal fees from Celgene, personal fees from Merck, personal fees from Novartis, personal fees from Roche, personal fees from Sanofi-Genzyme, outside the submitted work.

CPK has nothing to disclose.

PM has nothing to disclose.

MT reports grants, personal fees, and nonfinancial support from Roche, grants, personal fees and nonfinancial support from Biogen, grants, personal fees, and nonfinancial support from Merck, grants, personal fees, and nonfinancial support from Sanofi, grants and personal fees from Novartis, outside the submitted work.

\section{Statement of Authorship}

APL, GP, TB, AR, RDP, CPK, PM, and MT participated in the concept, design, analysis, writing, and revision of the manuscript.

\section{REFERENCES}

1. International Multiple Sclerosis Genetics Consortium, Wellcome Trust Case Control Consortium, Sawcer S, et al. Genetic risk and a primary role for cell-mediated immune mechanisms in multiple sclerosis. Nature. 2011;476(7359):214-9.

2. Moutsianas L, Jostins L, Beecham AH, et al. Class II HLA interactions modulate genetic risk for multiple sclerosis. Nat Genet. 2015;47(10):1107-13

3. Link J, Kockum I, Lorentzen AR, et al. Importance of human leukocyte antigen (HLA) class I and II alleles on the risk of multiple sclerosis. PLoS One 2012;7(5):e36779.

4. Oksenberg JR, Barcellos LF, Cree BA, et al. Mapping multiple sclerosis susceptibility to the HLA-DR locus in African Americans. Am J Hum Genet. 2004;74(1):160-7.
5. Dyment DA, Herrera BM, Cader MZ, et al. Complex interactions among MHC haplotypes in multiple sclerosis: susceptibility and resistance. Hum Mol Genet. 2005;14(14):2019-26.

6. Zhang Q, Lin CY, Dong Q, Wang J, Wang W. Relationship between HLA-DRB1 polymorphism and susceptibility or resistance to multiple sclerosis in Caucasians: a meta-analysis of non-familybased studies. Autoimmun Rev. 2011;10(8):474-81.

7. Lincoln MR, Montpetit A, Cader MZ, et al. A pre- dominant role for the HLA class II region in the association of the MHC region with multiple sclerosis. Nat Genet. 2005;37(10):1108-12.

8. Bergamaschi L, Leone MA, Fasano ME, et al. HLA-class I markers and multiple sclerosis susceptibility in the Italian population. Genes Immun. 2010;11(2):173-80.

9. Brynedal B, Duvefelt K, Jonasdottir G, et al. HLA- A confers an HLA-DRB1 independent influence on the risk of multiple sclerosis, PLoS One. 2007;2(7):e664.

10. Fogdell-Hahn A, Ligers A, Gronning M, Hillert J, Olerup O. Multiple sclerosis: a modifying influence of HLA class I genes in an HLA class II associated autoimmune disease. Tissue Antigens. 2000;55(2):140-8

11. Healy BC, Liguori M, Tran D, et al. HLA B*44: protective effects in MS susceptibility and MRI outcome measures. Neurology. 2010;75(7):634-40.

12. Silva AM, Bettencourt A, Pereira $\mathrm{C}$, et al. Protective role of the HLA-A*02 allele in Portuguese patients with multiple sclerosis. Mult Scler. 2009;15(6):771-4.

13. Hollsberg P, Hansen HJ, Haahr S. Altered CD8+ T cell responses to selected Epstein-Barr virus immunodominant epitopes in patients with multiple sclerosis. Clin Exp Immunol. 2003;132(1): 137-43.

14. Burfoot RK, Jensen CJ, Field J, et al. SNP mapping and candidate gene sequencing in the class I region of the HLA complex: searching for multiple sclerosis susceptibility genes in Tasmanians. Tissue Antigens. 2008;71(1):42-50.

15. Hensiek AE, Sawcer SJ, Feakes R, et al. HLA-DR 15 is associated with female sex and younger age at diagnosis in multiple sclerosis. J Neurol Neurosurg Psychiatry. 2002;72(2):184-7.

16. Liguori M, Healy BC, Glanz BI, et al. HLA (A- B-C and -DRB1) alleles and brain MRI changes in multiple sclerosis: a longitudinal study. Genes Immun. 2001;12(3):183-90.

17. Okuda DT, Srinivasan R, Oksenberg JR, et al. Genotype-phenotype correlations in multiple sclerosis: HLA genes influence disease severity inferred by 1HMR spectroscopy and MRI measures. Brain. 2009;132(Pt 1):250-9.

18. Ramagopalan SV, Maugeri NJ, Handunnetthi L, et al. Expression of the multiple sclerosis-associated MHC class II Allele HLADRB $1 * 1501$ is regulated by vitamin D. PLoS Genet. 2009;5(2): e1000369.

19. Chao MJ, Ramagopalan SV, Herrera BM, et al. Epigenetics in multiple sclerosis susceptibility: difference in transgenerational risk localizes to the major histocompatibility complex. Hum Mol Genet. 2009;18(2):261-6.

20. Celius EG, Harbo HF, Egeland T, Vartdal F, Vandvik B, Spurkiand A. Sex and age at diagnosis are correlated with the HLA-DR2, DQ6 haplotype in multiple sclerosis. J Neurol Sci. 2000;178(2):132-5.

21. DeLuca GC, Ramagopalan SV, Herrera BM, et al. An extremes of outcome strategy provides evidence that multiple sclerosis severity is determined by alleles at the HLA-DRB1 locus. Proc Natl Acad Sci U S A. 2007;104(52):20896-901.

22. McDonnell GV, Mawhinney H, Graham CA, Hawkins SA, Middleton D. A study of the HLA-DR region in clinical subgroups of multiple sclerosis and its influence on prognosis. J Neurol Sci. 1999;165(1):77-83.

23. Lysandropoulos AP, Mavroudakis N, Pandolfo M, et al. HLA genotype as a marker of multiple sclerosis prognosis: a pilot study. J Neurol Sci. 2017;15(375):348-54.

24. Jain S, Sima DM, Ribbens A, et al. Automatic segmentation and volumetry of multiple sclerosis brain lesions from MR images. Neuroimage Clin. 2015;16(8):367-75.

25. Smeets D, Ribbens A, Sima DM, et al. Reliable measurements of brain atrophy in individual patients with multiple sclerosis. Brain Behav. 2016;6(9):e00518. 
26. Jain S, Ribbens A, Sima DM, et al. Two time point MS lesion segmentation in brain MRI: an expectation-maximization framework. Front Neurosci. 2016;10:576.

27. Zivadinov R, Uxa L, Zacchi T, et al. HLA genotypes and disease severity assessed by magnetic resonance imaging findings in patients with multiple sclerosis. J Neurol. 2003;250(9):1099-106.

28. Zivadinov R, Weinstock-Guttman B, Zorzon M, et al. Geneenvironment interactions between HLA B7/A2, EBV antibodies are associated with MRI injury in multiple sclerosis. J Neuroimmunol. 2009;209(1-2):123-30.

29. McFarland HF, Martin R. Multiple sclerosis: a complicated picture of autoimmunity. Nat Immunol. 2007;8(9):913-9.

30. Johnson AJ, Suidan GL, McDole J, Pirko I. The CD8 T cell in multiple sclerosis: suppressor cell or mediator of neuropathology? Int Rev Neurobiol. 2007;79:73-97. 